\title{
Platinum Silicide-Silicon Schottky Diode Characteristics
}

\section{S.N. Musaeva ${ }^{1}$ E.A. Kerimov ${ }^{2 *}$ N.F. Kazımov ${ }^{3}$ S.I. Huseynova ${ }^{3}$}

1. Azerbaijan Technical University, Baku, AZ1073, Azerbaijan

2. War College of Armed Forces, Azerbaijan Republic Ministry of Defense, Baku, AZ1000, Azerbaijan

3. Institute of Ecology, National Aerospace Agency, Baku, AZ1000, Azerbaijan

*Corresponding Author: E.A. Kerimov, War College of Armed Forces, Azerbaijan Republic Ministry of Defense, Baku, AZ1000, Azerbaijan. E-mail: e_kerimov.fizik@mail.ru

DOI: http://dx.doi.org/10.26549/met.v2i2.850

\section{Introduction}

$\mathrm{P}$ hotoelectric measurements are used to obtain additional information on the transportation mechanism of Schottky barrier structures.

The main purpose of the study is to investigate the photoelectric properties of Shottky diodes based on PtSi - n Si and PtSi - p - Si contacts.

For the purpose of the study, two types of Schottky diodes were developed using the method described in. ${ }^{[1]}$ The first type of device is on $\mathrm{n}-\mathrm{Si}$ (Figure 1), and the second type is on p-Si (Figure 2 (a),(b)).

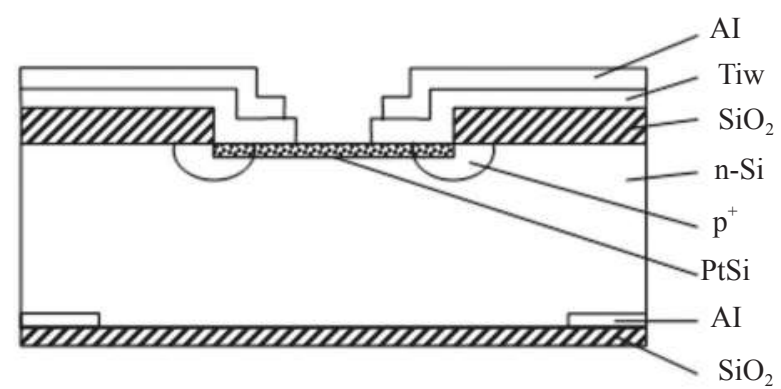

Figure 1. Schematic Picture of Schottky Barrier Structure on PtSi-n-Si

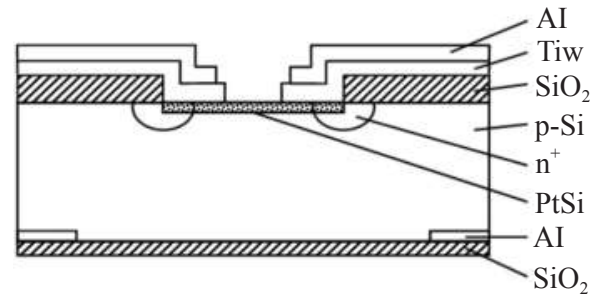

Figure 2 (a). Schematic Picture of Schottky Barrier Structure on PtSi-n-Si (In this case, ohmic contact is Al ring-shaped form)

Silicone p-type Al-contact is a single ring-shaped (Figure 2 (a)), and in the other case it is whole (Figure 2 (b)).

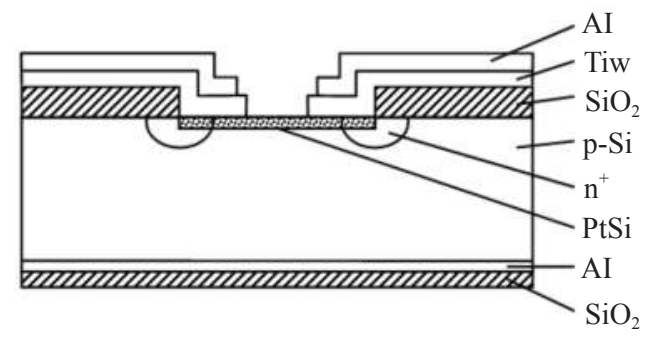

Figure 2 (b). Schematic Picture of Schottky Barrier Structure on PtSi-n-Si (In this case, omic contact $\mathrm{Al}$ is intact) 
The technology of getting both of the structures is the same.

As a result of the thermal processing of the platinum, the platinum is processed by platinum silicide ( $\mathrm{PtSi})$, which is metallic silicide, not semiconductor, but with a MnP-type orthomorphic structure and cage constants $\mathrm{a}=$ $5,59 \mathrm{~A}^{0}, \mathrm{~b}=3,603 \mathrm{~A}^{0}, \mathrm{c}=5,932 \mathrm{~A}^{0}$. ${ }^{[1]}$

The Schottky barrier appears in the silica-platinum silicide contact and PtSi plays role the of metal.

The role of ohmic contact is Aluminum (Al).

Titanium voltage (TiW) plays the role of the diffusion layer,which prevents the diffusion of the Al-ohmic contact to PtSi.

The structures that we have prepared are devastated the thickness of PtSi from existing structures, so that, the method described in ${ }^{[2]}$ allows the thickness of PtSi to be reduced to $100 \mathrm{~A}^{0}$.

For photodiodes operating in the infrared region (Figure 2(a),(b)), the smaller the thickness of PtSi, the greater the swelling of infrared rays in $\mathrm{PtSi}$, according to the classical Drude theory.

\section{Experiment}

Figure 3 shows the experimental dependence of the PtSi n - Si structure sensitivity.

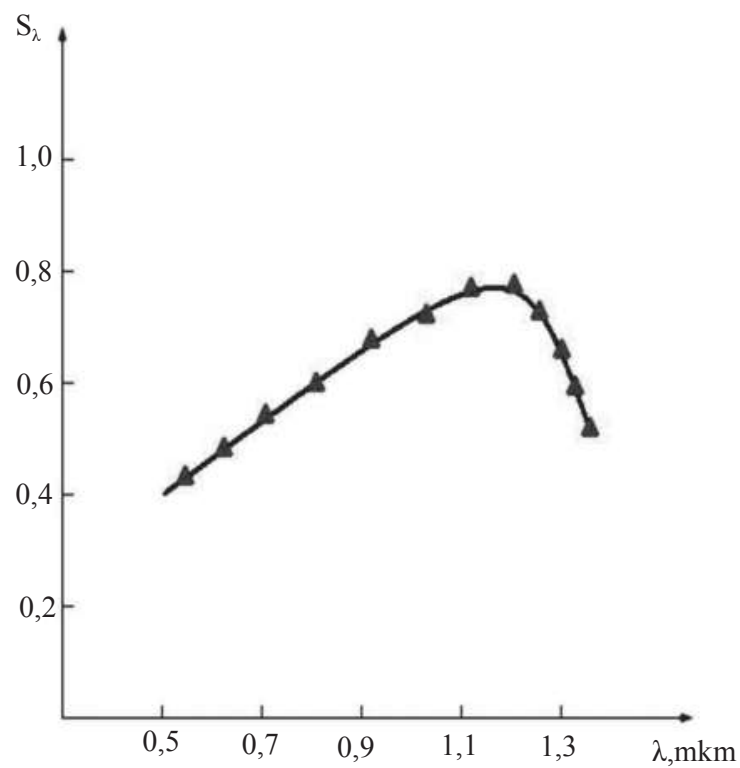

Figure 3. Spectral Characteristics of Schottky Barrier Structures Based on PtSi-n-Si Contact.(Values in Figure 3 are presented with commas)

The spectral property of the build increases at the beginning,and then reaches its maximum value at a value of $1,125 \mathrm{mkm}$ in the wavelength and reduces again.

The maximal state of the photocurrent is suitable for the main swallowing of silicon, that is conditioned by the generation of electron- hole pairs in photoconductivity .

The increase in longwave border is related to the photoemission of the electron from metal to silicon.

This long wave border of spectral characteristic of photoelements is determined by the height of the potential fence.

The height of the barrier for PtSi $-\mathrm{n}-\mathrm{Si}$ structure is $0.830 .94 \mathrm{eV}$, and the red border of the photoeffect comply with $1,321,49 \mathrm{mkm}$.

The height of the barrier was $0.93 \mathrm{eV}$ in our case, so the red border of the photoeffect corresponds to $1.3 \mathrm{mkm}$. PtSi - p - Si structures (Fig. 2) show a case study of the reflective volt - amp (VAX) characteristics, whether or not they have light that, varies considerably depending on the voltage reflected during the lighting, while the opposite voltage is almost 103 times higher when it is $6 \div 8 \mathrm{~V}$, ie the optimal operating mode of PtSi - p - Si Schottky barrier structures is $\mathrm{Ur}=6 \div 8 \mathrm{~V}$.

In the case of large currents, the cost of currents increases and the diodes deteriorate in the specimens examined.(IKC - 14 A spectrophotometry was used as light source, and light on the studied PtSi was dropped from silicon side).

The spectral distribution of temperature sensitivity $\mathrm{T}=$ $800 \mathrm{~K}$ is described in PtSi - p - Si structure. (Figure 4)

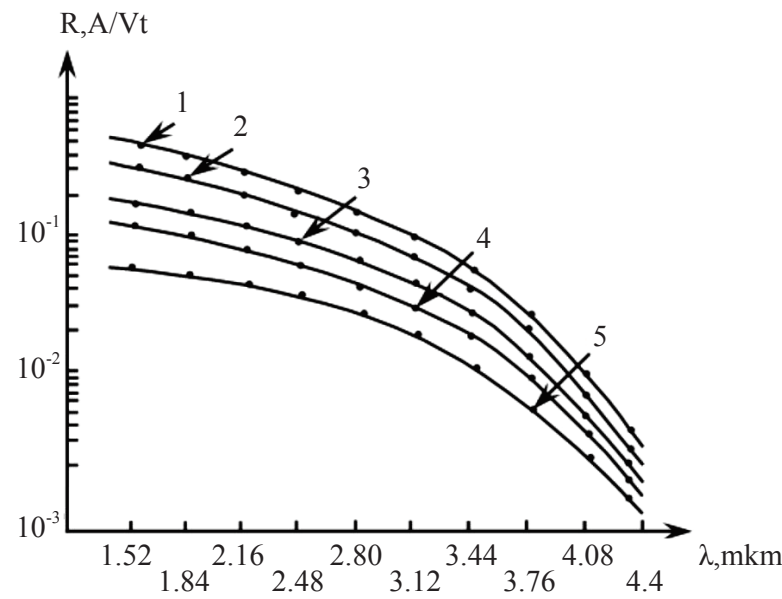

Figure 4. Spectral Characteristics of PtSi-p-Si Structures at Different Thicknesses of PtSi: 1-100 A ${ }^{0}, 2-300 A^{0}, 3$ $400 \mathrm{~A}^{0}, 4-500 \mathrm{~A}^{0}, 6-600 \mathrm{~A}^{0}$

\section{Explanation of Results}

The photodiode illustrated in Figure 1 works in the visible region,there is no need to explain the principle of working.

The diodes described in Figure 2 and Figure 3 work in the infrared region.

In figure 5 is shown the energy diagram showing the 
work of Schottky photodiodes based on PtSi - p - Si.

Metal and semiconductor contacts can transition from metal to semiconductor or vice versa depending on the ratio of the electrons in the metal to the Fm-output and the ratio of the electrons to the semiconductor output, as a result of the internal emission.

In this case, the electrons pass from semi-conductor ( $\mathrm{Si}$ ) semiconductors to metal (silicon) and metal and also from Fermi levels to thermodynamic equilibrium.

Two types of electronic transitions may be possible in these structures thanks to infrared radiation.

If the energy of the falling photon $\mathrm{h} V \geq \mathrm{Eg}$ so, in the semiconductor, the generation of the electron - hole pair occurs when the photon is absorbed.

In this case, as in the conventional $\mathrm{p}-\mathrm{n}$ transition photodiode, the separation of different loaded carriers occurs, and the photo electric force is generated.

Obviously, the longitudinal limit of such a process can not be less than the inhibiting zone of the semiconductor, and Schottky barrier photodiodes are not different from photodiodes or specific photoreceptors.
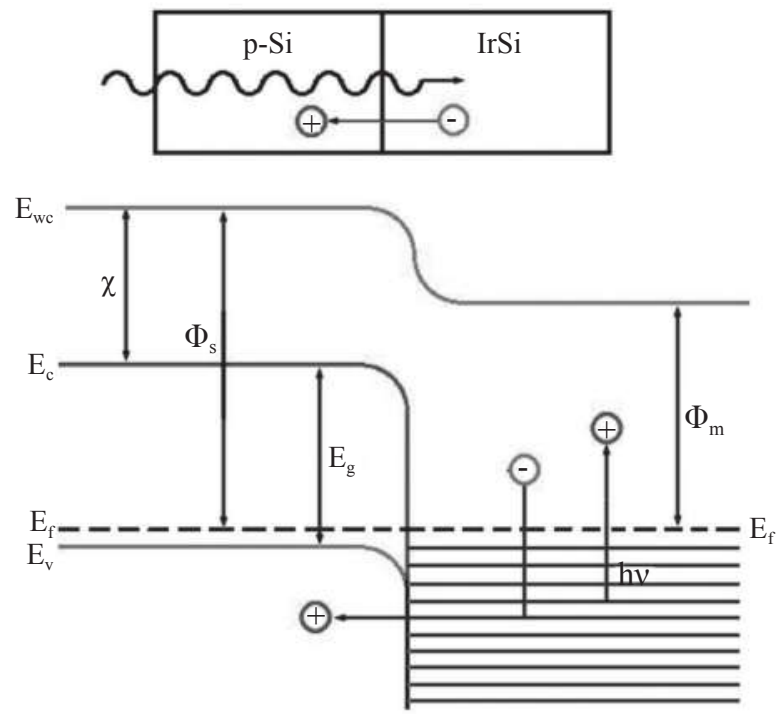

Figure 5. Energy Diagram Showing Schottky Photodiode Operation Based on Irsi (PtSi)-p-Si.

If $\mathrm{h} \boldsymbol{V}>\mathrm{Eg}$ the absorption of infrared radiation in the metal layer causes the valence electrons to be higher than the Fermi level, leading to the formation of holes that some of these holes have greater energy than the barrier of $\psi \mathrm{ms}$.

Subsequently, the hole emits either hollow metal (in our case from PtSi) to the semiconductor, or by emptying the semiconductor to the metal by filling the empty state. $\mathrm{PtSi}$ - p - Si contact potential holes with high energy holes will pass to semiconductor.

Increase of $\mathrm{h} \boldsymbol{V}$ or the number of holes in the light wave length decreases

This will create a photocurrent

So the basis for p-type silicon loaders are considered holes.

For circular and integral planar contact structures, the cost of the quench photoemiss coefficient

$(\mathrm{Yh} \boldsymbol{V})^{1 / 2}=\mathrm{cl}(\mathrm{h} \boldsymbol{V}-\psi)$ is determined from the inclination angle of $1 / 2=\mathrm{c} 1(\mathrm{~h} \boldsymbol{V}-\psi)$ and the corresponding strike values are 0.126 and $0.048 \mathrm{eV}-1$.

Here $\mathrm{Y}$ - is the improvement factor.

The quantum emission coefficient remains constant for non-circular contacts with a reduction in PtSi thickness from $600 \mathrm{~A}^{0}$ to $100 \mathrm{~A}^{0}$.

The spectral characteristic of the circular contact structures is shown in Figure 4, where absolute photoperiod is increased with the decrease in PtSi thickness.

To simulate experimental results, a simplified one-dimensional model for the photocurrent of Schottky barrier photodiode has been proposed. ${ }^{[3]}$

This model suggests ideal visibility in the silicon - insulator and silicon - semiconductor boundary, except for the holes in the holes in the silicon and the ideal release on the silicon - metal boundary

In this model, the Wi - Factor included that it is likely that the hot luminaire, ie energy, which is greater than the height of the potential barrier, may reach from one separation boundary to another, which is the function of the average length of silicon thickness and freezing path of the holes:

$\mathrm{Wi}=\exp (-\mathrm{d} / \mathrm{L})$

Here is $\mathrm{d}$ - the thickness of PtSi, L is the length of the free running path of the holes.

Then the factor of improvement is the proportion of the photographer, expressed in the following formula:

$\mathrm{Y}=\mathrm{Y}_{1} / \mathrm{Y}_{2}=1 /(1-\exp (-\mathrm{d} / \mathrm{L})$

As a result of calculating $d=100 \mathrm{~A}^{0}$ and $\mathrm{Y}=5$, the average length of free running path of hot holes was $L=430$ $\mathrm{A}^{0}$.

\section{References}

[1] Sh.Myurarka. Silicides for VLSI.Moscow «МИР»1986, Translated from English by Candidate of Technical Sciences V.V. Baranova, under the title of Prof., Dr.Sc. Yu.D.Chistyakov. (in Russian)

[2] Kerimov E.A, Guluzade B.A, Hasanova R.R. Pd - Si Structures Acquisition Technology and Photoelectric Properties.Physics.2009, p.15, No. 4, pp. 35-37. (in Russian)

[3] E.A. Kerimov .Photosensitive element based on the İrSi Si contact.News of ANAS, Physics and Astronomy 2011, №5, Volume XXXI, p.158-161. (in Russian) 